\section{Case Reports in Ophthalmology}

Case Rep Ophthalmol 2020;11:242-248

DOI: $10.1159 / 000508846$

Published online: June 29, 2020
(C) 2020 The Author(s)

Published by S. Karger AG, Basel www.karger.com/cop

This article is licensed under the Creative Commons Attribution-NonCommercial 4.0 International License (CC BY-NC) (http://www.karger.com/Services/OpenAccessLicense). Usage and distribution for commercial purposes requires written permission.

\title{
Small Symptomatic Unilateral Choroidal Osteoma Diagnosed with Enhanced-Depth Imaging Optical Coherence Tomography and Thin-Slice Computed Tomography: 2 Clinical Cases
}

\author{
Hiroko Suzuki ${ }^{a}$ Ryo Obata ${ }^{a}$ Xue Tan ${ }^{a}$ Ryo Terao ${ }^{a}$ Keiko Azuma \\ Tatsuya Inoue $^{a}$ Shigenobu Suzuki ${ }^{b}$ \\ aDepartment of Ophthalmology, Graduate School of Medicine and Faculty of Medicine, \\ The University of Tokyo, Tokyo, Japan; ${ }^{b}$ Department of Ophthalmic Oncology, National \\ Cancer Center Hospital, Tokyo, Japan
}

\section{Keywords}

Choroidal osteoma - Enhanced-depth optical coherence tomography · Computed tomography

\begin{abstract}
Background: Choroidal osteoma is a rare, benign, ossifying intraocular tumor of unknown etiology. While patients with choroidal osteoma usually show distinct large yellowish subretinal lesions, some could have small lesions, making the differential diagnosis difficult. We experienced 2 cases of small symptomatic unilateral osteoma approximately $1.0-\mathrm{mm}$ disc diameter in size. Methods: Retrospective medical charts of 2 patients with small symptomatic unilateral osteoma were reviewed. Fundus examination, spectral domain enhanced-depth optical coherence tomography (EDI-OCT), fluorescein angiography, indocyanine green angiography, B-scan ultrasonography (USG), and X-ray computed tomography (CT) were performed. Results: Case 1: a case of a 41-year-old male. Fundus examination revealed a yellowish-white lesion of 1.0$\mathrm{mm}$ disc diameter in size. EDI-OCT of the lesion shows sub-RPE elevation. B-scan USG was not definite for diagnosis. Thin-slice $(2 \mathrm{~mm})$ CT scan revealed a choroidal osteoma. Case 2: a case of a 70-year-old male. Fundus examination revealed a yellowish-white lesion of 0.9-mm disc
\end{abstract}


diameter. EDI-OCT showed sub-RPE elevation without serous retinal detachment and horizontal lamellar-like structure within the lesion. B-scan USG suggested acoustic shadowing, but it was unclear. The thin-slice CT scan confirmed bony tissue at the lesion. Conclusions: We report 2 cases of small choroidal osteoma. Diagnosis of small yellowish subretinal lesions is sometimes difficult. Characteristic findings with EDI-OCT may sometimes suggest this disease, and thin-slice CT could help to diagnose choroidal osteoma.

(C) 2020 The Author(s)

Published by S. Karger AG, Basel

\section{Introduction}

Choroidal osteoma is a rare, benign, ossifying intraocular tumor, first described in 1979 by Gass et al. [1]. They are classically present in females in their second or third decade of life, but sometimes found in the aged population [2]. Despite its benign nature, choroidal osteoma may cause severe visual loss due to pigment epithelium atrophy or serosanguinous changes with or without choroidal neovascularization [3, 4].

The tumor is generally unilateral and located in the juxtapapillary or macular region [5]. While patients with choroidal osteoma usually show distinct large yellowish subretinal lesions, some could have small lesions. This would make the differential diagnosis difficult because several pathologies have similar fundoscopic findings.

We experienced 2 cases of small choroidal osteoma presenting as a yellowish subretinal lesion of approximately 1.0-mm disc diameter in size. In one case intralesional structure with optical coherence tomography (OCT) suggested choroidal osteoma, and thin-slice computed tomography (CT) of both cases helped to differentiate from other diseases.

\section{Case Report/Case Presentation}

\section{Patients and Methods}

Two eyes of 2 consecutive patients with unilateral choroidal osteoma who had presented from January 1, 2015, to December 31, 2018, at the University of Tokyo Hospital were included in the study.

Both patients underwent comprehensive ophthalmic examinations including the measurement of best-corrected visual acuity (BCVA), slit-lamp biomicroscopy, funduscopy, and spectral domain enhanced-depth OCT (Heidelberg Spectralis ${ }^{\circledR}$, Heidelberg, Germany). Fluorescein angiography (FA) and indocyanine green angiography (ICGA) were performed using a Heidelberg Retina Angiograph II ${ }^{\circledR}$ (Heidelberg, Germany). B-scan ultrasonography (USG) was performed using UD-8000 (Tomey, Nagoya, Aichi). Because the USG findings were not definite for diagnosis, 1- or 2-mm slice X-ray CT of the orbit was also performed.

\section{Case Presentation}

Case 1

A 41-year-old male presented to an eye clinic with metamorphopsia and reduced vision in the right eye in 2011. At the initial examination, BCVA in his right eye was 20/20. Fundus examination revealed a whitish lesion in the macula and OCT showed serous retinal detachment (SRD) accompanied with a fibrin-like deposit. He was initially diagnosed with central serous chorioretinopathy (CSC). Five months after the initial visit, BCVA worsened to 16/20. OCT revealed the recurrence of SRD and macular edema. According to laboratory data, 
infectious or noninfectious inflammatory diseases were not suggested. Because solitary idiopathic choroiditis (SIC) [6]. was suspected, oral prednisolone was administered for over 2 months, though the condition did not change.

He was then referred to our clinic. Slit-lamp anterior segment examination was unremarkable, and the intraocular pressure was normal. Fundus examination revealed a subretinal yellowish-white lesion of 1.0-mm disc diameter in size between the fovea and the optic disc (Fig. 1). OCT scan disclosed SRD and intraretinal fluid extending to the foveal center, and a dome-like sub-RPE elevation corresponding to the yellowish lesion. There was shallow detachment of the RPE. Beneath the RPE, horizontal lamellar-like structure was seen within the choroid. Bruch's membrane and the sclera-choroidal junction around the lesion was detectable. The choriocapillaris and part of the choroidal vessels were not visible within the lesion (Fig. 1). FA revealed staining of the plaque in the late phase of the angiogram, and ICGA revealed hypofluorescence in the early phase and hyperfluorescence in the late phase (online suppl. Fig. S1; for all online suppl. material, see www.karger.com/doi/10.1159/000508846). B-scan USG suspected a thin hypoechoic shadow, but it was not definite for diagnosis. We performed thin-slice $(2 \mathrm{~mm})$ CT scan of the orbit. It revealed that the lesion had the density of bony tissue, leading to the diagnosis of a choroidal osteoma (online suppl. Fig. S2). Four years after the initial visit, mild subretinal hemorrhage developed which was associated with the lesion. OCT angiography shows a vascular network adjacent to the tumor.

Case 2

A 70-year-old male was referred to our clinic with fundoscopic abnormality in the left eye in 2014 . His visual acuity was $25 / 20$ in both eyes on examination at our hospital. Slit-lamp anterior segment examination and intraocular pressures were normal in both eyes. Fundus examination revealed a yellowish-white lesion of 0.9-mm disc diameter in the left eye (Fig. 2). OCT scan showed mild sub-RPE elevation corresponding to the yellowish lesion (Fig. 2). There was subtle detachment of the RPE. Intralesional structure beneath the RPE was unclear. Bruch's membrane and the sclera-choroidal junction at the lesion were detectable. The choriocapillaris and the inner part of the choroidal vessels were not visible. FA revealed staining of the plaque in the late phase of the angiogram, and ICGA revealed hypofluorescence in the early and late phase (online suppl. Fig. S3). B-scan USG in the left eye suspected a thin hypoechoic shadow, but it was not definite for diagnosis (Fig. 3). Thin-slice (1 mm) CT scan of the orbit was performed and showed the lesion had the density of bony tissue, confirming that the diagnosis was choroidal osteoma (Fig. 3). Three years after the initial visit, SRD developed around the lesion and the patient complained of blurred vision. The visual acuity remained at $25 / 20$. Choroidal neovascularization was not detected in angiography.

\section{Discussion/Conclusion}

\section{Discussion}

Most patients with choroidal osteoma are easily diagnosed with its characteristic findings. Shields et al. [7] described in a review that ophthalmoscopically the tumor usually ranges in size from approximately 2 to $22 \mathrm{~mm}$ in basal dimensions and from approximately 0.5 to 2.5 $\mathrm{mm}$ in elevation. In a retrospective study of 74 eyes with unilateral or bilateral choroidal osteoma the average tumor size at base was $8.2 \mathrm{~mm}$ (range, 1-20) [3]. Some could have small lesions, making the diagnosis difficult, but there have been few reports on small choroidal 


\section{Case Reports in Ophthalmology}

Case Rep Ophthalmol 2020;11:242-248

DOI: $10.1159 / 000508846$

c) 2020 The Author(s). Published by S. Karger AG, Basel www.karger.com/cop

Suzuki et al.: EDI-OCT and Thin-Slice CT for the Diagnosis of Choroidal Osteoma

osteoma. Herein we report 2 cases of small symptomatic unilateral choroidal osteoma approximately 1.0-mm disc diameter in size.

When the lesion is small, differential diagnosis may be difficult because several diseases show similar unilateral, orange-brown subretinal lesions. Diseases that need to be ruled out include amelanotic choroidal melanoma, amelanotic choroidal nevus, metastatic carcinoma, circumscribed choroidal hemangioma, age-related macular degeneration, CSC, organized subretinal hemorrhage, posterior scleritis, metastatic choroidal calcification, dystrophic choroidal calcification, choroidal granuloma, SIC [6], and sclerochoroidal calcification [8]. SIC is characterized by a solitary, yellowish-white choroidal mass of 1.0-mm disc diameter in basal dimension [6]. Administrating systemic corticosteroids is an effective treatment for SIC, especially when located in the region of the optic disc or the fovea [6].

In case 1, the patient demonstrated a yellowish-white lesion, and CSC or SIC was initially suspected. In both cases, echogram could not clearly show typical hyperacoustic signals because the lesion was too small. Thin-slice CT of the orbit, however, helped the diagnosis.

Recent studies reported characteristic findings in choroidal osteoma with spectral domain enhanced-depth OCT (EDI-OCT) [9, 10]. The findings included hyperreflective horizontal lamella, horizontal/vertical tubular lamella, or spongy lesion. Horizontal lamella was thought to be specific to choroidal osteoma. In case 1, EDI-OCT showed lamella-like intralesional structure that helped to suspect choroidal osteoma. In case 2 these findings were not clear probably because of the small size of the lesion. In contrast, thin-slice CT definitely showed calcification in the area corresponding to the lesion. Jokl et al. [11] reported usefulness of CT scanning for the detection of choroidal calcium in 2 patients with choroidal osteoma or pseudoxanthoma elasticum. We believe thin-slice CT should be considered when the diagnosis of subretinal yellowish lesion is not definite using other examinations.

There are several limitations in the present reports. First, in the present cases, the USG was performed through the patients' eyelids. However, if the scanning were made directly through the eyeball, it could improve the clarity of the image. Furthermore, A-scan (amplitude) tracing could have picked up high echoes at the lesion, helping the diagnosis. Considering CT scanning exposes the patient to radiation, it should be used only when the USG is not definite for the diagnosis. Second, it is true that CT scanning was helpful to detect the presence of calcium, but the lesion with calcification was not necessarily choroidal osteoma. Other diseases with calcification such as retinoblastoma or hemangioma must be ruled out using other imaging modalities before CT scanning. However, when the diagnosis is equivocal with other imaging modalities, CT scanning should be an option even for small lesions.

\section{Conclusion}

We report 2 cases of small choroidal osteoma of less than 1.0-mm disc diameter in size. Diagnosis of small yellowish subretinal lesion is sometimes difficult. Characteristic findings with EDI-OCT may sometimes suggest this disease, and thin-slice CT could help to differentiate between choroidal osteoma and other pathologies.

\section{Statement of Ethics}

In the current case series, we retrospectively reviewed the charts of patients in the outpatient clinic of the University of Tokyo Hospital. The study was in accordance with the tenets of the Declaration of Helsinki and was approved by the institutional review board of the 


\section{Case Reports in Ophthalmology}

University of Tokyo as a retrospective review of the patients' medical records. Written informed consent was not required in the study because the data was anonymously used.

\section{Disclosure Statement}

Ryo Obata has received honoraria from Novartis Pharma, Bayer, and Santen Pharmaceutical.

\section{Funding Sources}

The authors did not receive any financial support for this study.

\section{Author Contributions}

H.S., R.O., and S.S.: conception or design of the work and drafting the manuscript. H.S., R.O., and X.T.: acquisition and analysis of data. R.T., K.A., and T.I.: interpretation of data and critically revising the manuscript. All authors read and approved the final version of the manuscript for publication.

\section{References}

1 Gass JD, Guerry RK, Jack RL, Harris G. Choroidal Osteoma. Arch Ophthalmol. 1978 Mar;96(3):428-35.

2 Browning DJ. Choroidal osteoma: observations from a community setting. Ophthalmology. 2003 Jul;110(7):1327-34.

3 Shields CL, Sun H, Demirci H, Shields JA. Factors predictive of tumor growth, tumor decalcification, choroidal neovascularization, and visual outcome in 74 eyes with choroidal osteoma. Arch Ophthalmol. 2005 Dec;123(12):1658-66.

4 Song MH, Roh YJ. Intravitreal ranibizumab in a patient with choroidal neovascularization secondary to choroidal osteoma. Eye (Lond). 2009 Aug;23(8):1745-6.

5 Aylward GW, Chang TS, Pautler SE, Gass JD. A long-term follow-up of choroidal osteoma. Arch Ophthalmol. 1998 Oct;116(10):1337-41.

6 Shields JA, Shields CL, Demirci H, Hanovar S. Solitary idiopathic choroiditis: the Richard B. Weaver lecture. Arch Ophthalmol. 2002 Mar;120(3):311-9.

7 Shields CL, Shields JA, Augsburger JJ. Choroidal osteoma. Surv Ophthalmol. 1988 Jul-Aug;33(1):17-27.

8 Honavar SG, Shields CL, Demirci H, Shields JA. Sclerochoroidal calcification: clinical manifestations and systemic associations. Arch Ophthalmol. 2001 Jun;119(6):833-40.

9 Shields CL, Pellegrini M, Ferenczy SR, Shields JA. Enhanced depth imaging optical coherence tomography of intraocular tumors: from placid to seasick to rock and rolling topography-the 2013 Francesco Orzalesi Lecture. Retina. 2014 Aug;34(8):1495-512.

10 Pellegrini M, Invernizzi A, Giani A, Staurenghi G. Enhanced depth imaging optical coherence tomography features of choroidal osteoma. Retina. 2014 May;34(5):958-63.

11 Jokl DH, Tsai N, Kreps S. Detection of choroidal calcium by computed tomography scan quantitative computerized tomography: a clinically useful diagnostic tool. Ophthalmology. 1999 Sep;106(9):1841-6. 


\section{Case Reports in Ophthalmology}
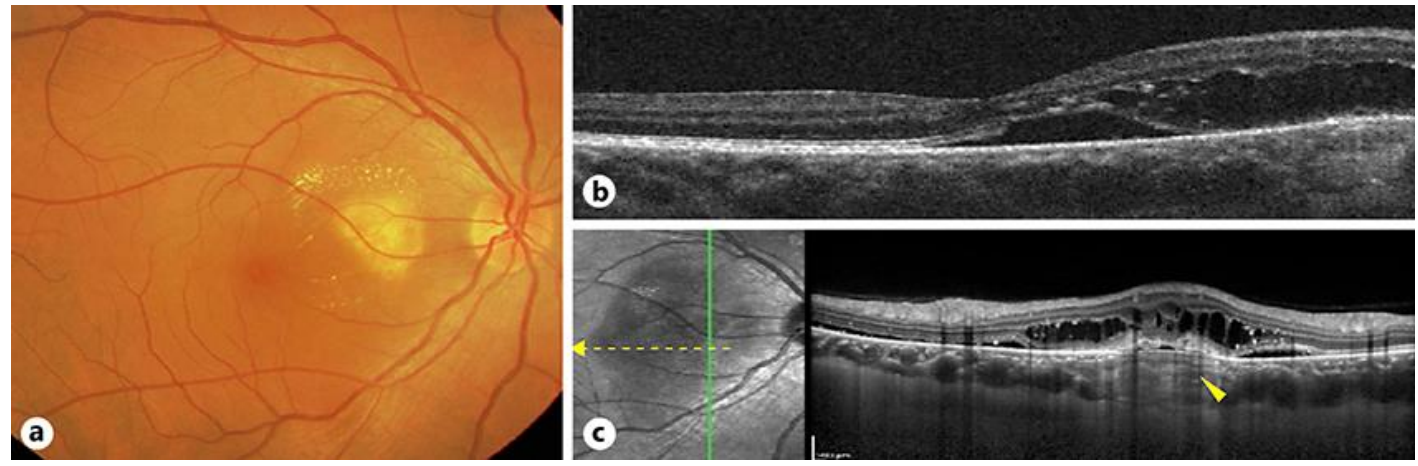

Fig. 1. Case 1. a A yellowish-white lesion of 1.0-mm disc diameter in size is located between the fovea and the optic disc. b OCT scan of the foveal center (yellow dashed line in c) disclosed serous retinal detachment (SRD) extending to the foveal center and intraretinal fluid. c OCT scan of the lesion (green solid line) shows sub-RPE mass with SRD. Lamella-like intralesional structure was seen within the mass (arrowhead).
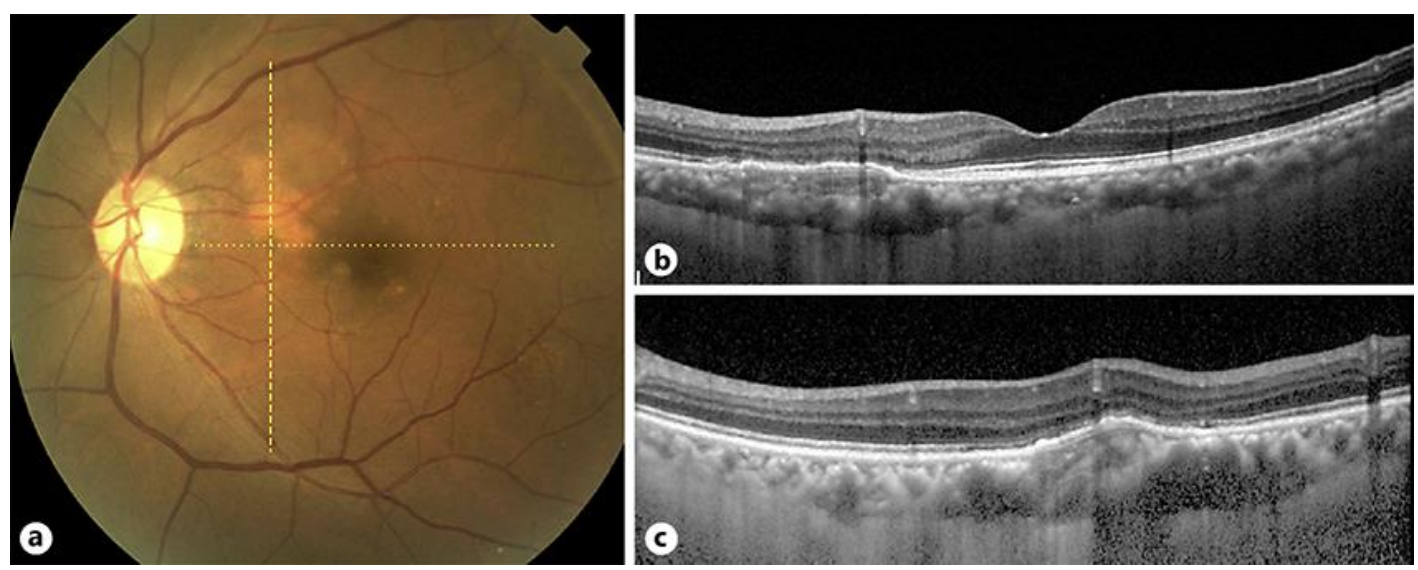

Fig. 2. Case 2. a A yellowish-white lesion of 0.9-mm disc diameter in size was located between the fovea and the optic disc. $\mathbf{b}$ OCT scan of the foveal center (horizontal dotted line in a) revealed shallow elevation of the RPE. c OCT scan of the lesion (vertical dashed line in a) showed sub-RPE mass. 
Case Reports in Ophthalmology
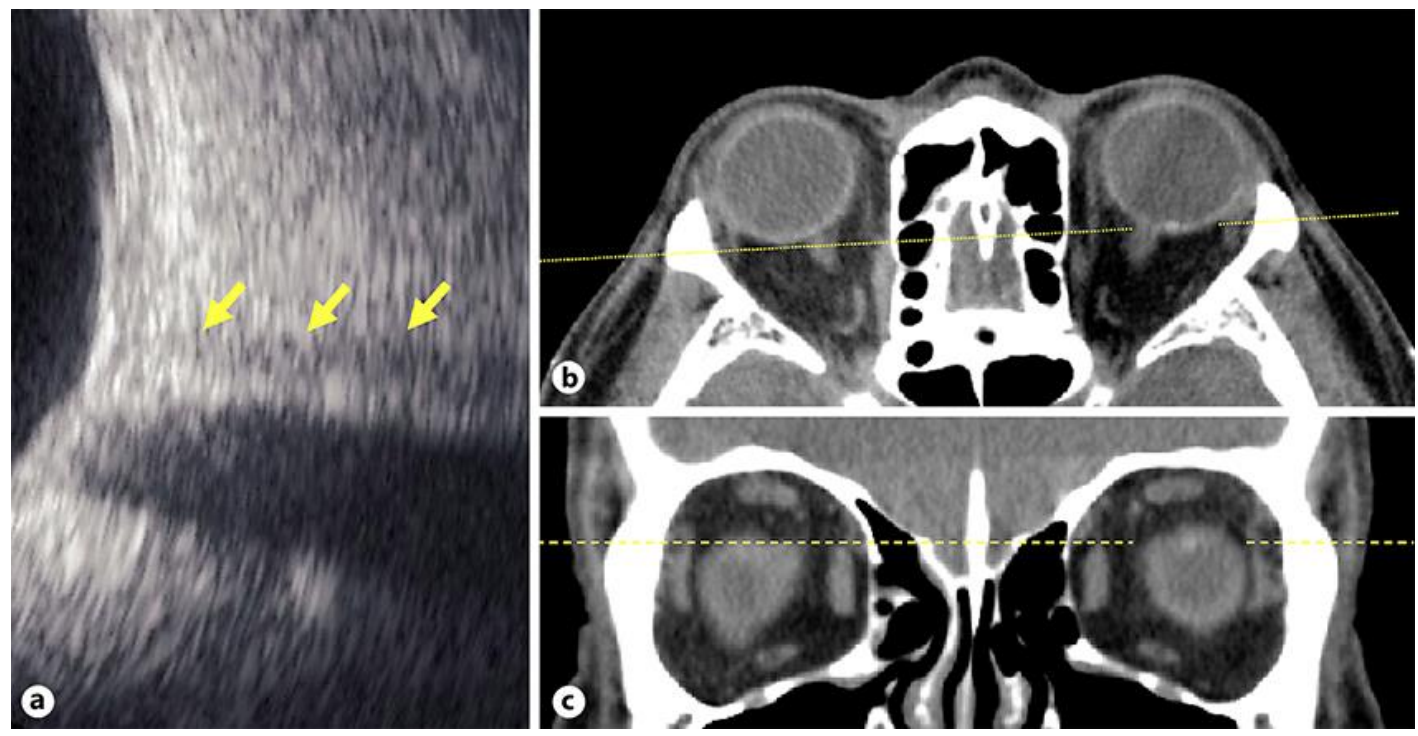

Fig. 3. Case 2. a B-scan USG in the left eye suspected acoustic shadowing (arrows). b Thin-slice axial CT revealed small lesion with density of bony tissue (dotted line indicates section plane of $\mathbf{c}$ ). $\mathbf{c}$ The coronal CT scan also revealed the lesion (dashed line indicates the section plane of $\mathbf{b}$ ). 